\title{
Case Report of E-cigarette Associated Lung Injury in a Health Female
}

\author{
Mario Fonseca-Paricio, MD \\ Thomas Jefferson University, mario.fonseca-paricio@jefferson.edu
}

Alan Gandler, MD

Thomas Jefferson Univeristy, alan.gandler@jefferson.edu

Boyd Hehn, MD

Thomas Jefferson University, Boyd.Hehn@jefferson.edu

Follow this and additional works at: https://jdc.jefferson.edu/tmf

Part of the Internal Medicine Commons

Let us know how access to this document benefits you

\section{Recommended Citation}

Fonseca-Paricio, MD, Mario; Gandler, MD, Alan; and Hehn, MD, Boyd (2020) "Case Report of E-cigarette Associated Lung Injury in a Health Female," The Medicine Forum: Vol. 21 , Article 14.

DOI: https://doi.org/10.29046/TMF.021.1.013

Available at: https://jdc.jefferson.edu/tmf/vol21/iss1/14

This Article is brought to you for free and open access by the Jefferson Digital Commons. The Jefferson Digital Commons is a service of Thomas Jefferson University's Center for Teaching and Learning (CTL). The Commons is a showcase for Jefferson books and journals, peer-reviewed scholarly publications, unique historical collections from the University archives, and teaching tools. The Jefferson Digital Commons allows researchers and interested readers anywhere in the world to learn about and keep up to date with Jefferson scholarship. This article has been accepted for inclusion in The Medicine Forum by an authorized administrator of the Jefferson Digital Commons. For more information, please contact: JeffersonDigitalCommons@jefferson.edu. 


\title{
Case Report of E-cigarette Associated Lung Injury in a Healthy Female
}

\author{
Mario Fonseca-Paricio, MD, Alan Gandler, MD, Boyd Hehn, MD
}

\section{CASE DESCRIPTION}

This is a case of a woman presenting with four days of diarrhea and dyspnea found to have E-cigarette or vapingassociated lung injury (EVALI).

The patient is a 30-year-old woman who presented to the hospital with a chief complaint of dyspnea on exertion. She developed dyspnea seven days prior to presentation; it was minimal at rest, however symptoms worsened with exertion. Her symptoms included fevers, intermittent chills, night sweats, dry persistent cough, and four days of profuse, watery diarrhea. She did not have sinus pain or congestion, headaches, or sore throat. Due to bloody diarrhea she sought medical care.

The patient had no allergies and took no medications regularly. She had a history of exercised-induced asthma during childhood that had resolved. Surgical history was notable for seven surgeries for bilateral breast fibroadenomas. Family history was notable for BRCA-negative breast cancer in her mother

She works as a real estate agent, with no known specific exposures. Ten days prior to admission, she had spent time with her nephew who she reported as having several symptoms consistent with an upper respiratory infection. She does not smoke cigarettes. She had been using electronic cigarettes with marijuana for 6 months earlier in the year, however due to news reports, she had recently stopped. She would smoke marijuana from electronic cigarettes four days a week with cartridges lasting about one week. Patient used no other drugs or alcohol. No history of homelessness or incarceration. Patient is sexually active with one male partner with no prior HIV or sexually transmitted infections (STI). On review of systems, patient noted to have an unintentional 5-pound weight loss over the past one week.

On initial vital signs, she had temperature of 38.2 C, HR of $117 \mathrm{bpm}$, blood pressure of 108/74 mmHg, respiratory rate of 22 breaths/minute, $86 \%$ on ambient air and $95 \%$ on 2 liters nasal cannula of oxygen. She was admitted to the medical service due to a new oxygen requirement.

Physical exam was notable for of a well-developed and nourished adult female in no acute distress on nasal cannula able to speak in full sentences. Pulmonary exam showed scattered rales with no wheezing. She had tachycardia with no jugular venous distention. She had no abdominal pain or lower extremity swelling.
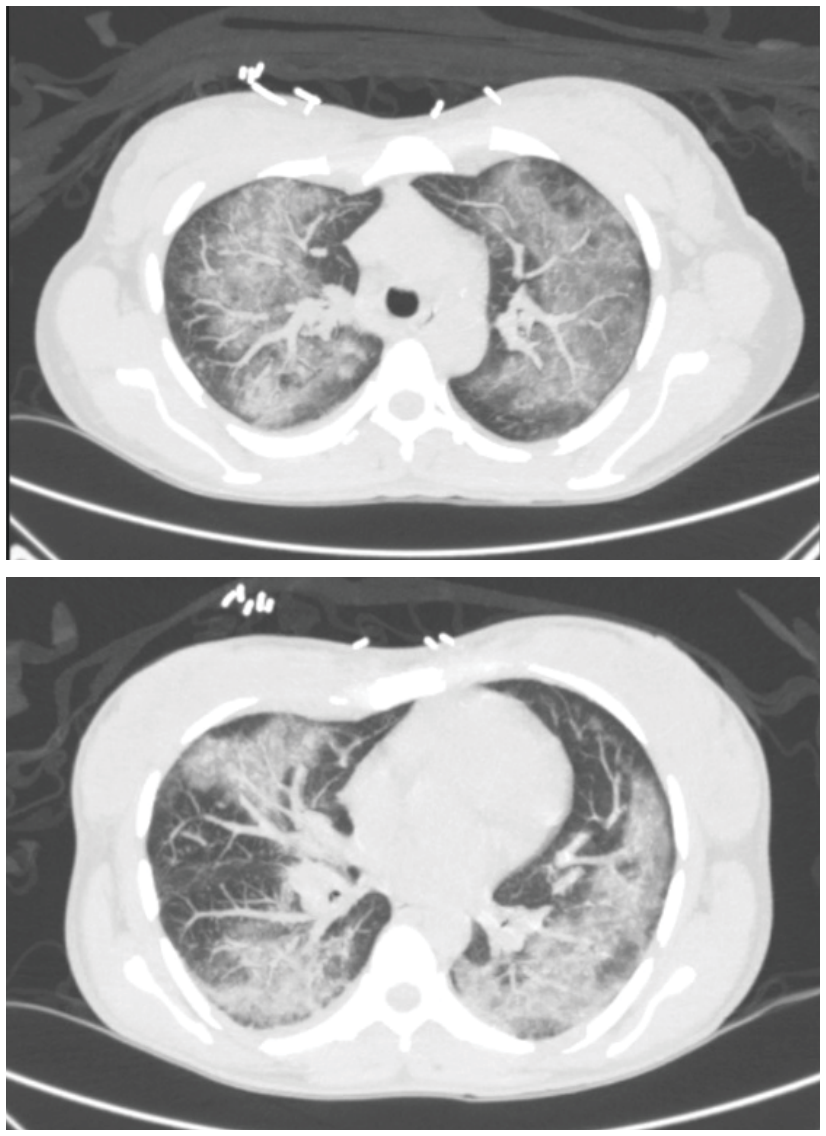

Figure 1. High-resolution CT of the chest demonstrating diffuse ground-glass opacities with septal thickening. There is a peripheral predilection with subpleural sparring disease.

Laboratory results on presentation revealed a leukocytosis of 28,000 cells $/ \mathrm{mm} 3$ with $92 \%$ neutrophils and a platelet count of 558 cells $/ \mathrm{mm} 3$. Electrolytes revealed a potassium of $2.9 \mathrm{mEq} / \mathrm{L}, \mathrm{HCO} 3$ of $22 \mathrm{mEq} / \mathrm{L}$. Liver function tests showed an AST/ALT of 119/73 IU/L. Lipase was normal. Lactate normal at $1.2 \mathrm{mmol} / \mathrm{L}$. Procalcitonin was $0.64 \mathrm{ng} / \mathrm{mL}$ (normal < $0.08 \mathrm{ng} / \mathrm{mL}$ )

Respiratory pathogen panel nasopharynx swab detected human rhinovirus/enterovirus on PCR. Blood cultures showed no growth. Influenza A/B, RSV PCR nasal swab was negative. Clostridium difficile toxin DNA, and gastrointestinal stool infectious panel were negative. HIV antibody and antigen combination were non-reactive. Streptococcus pneumoniae and Legionella antigen urine 

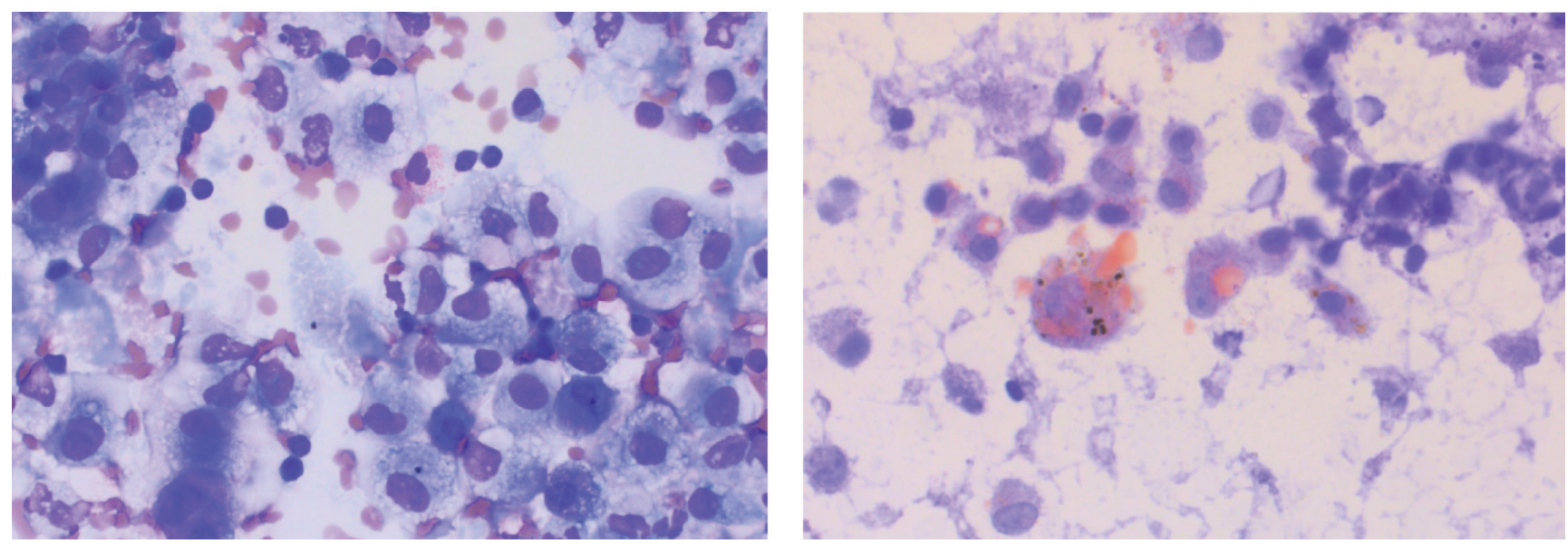

Figure 2. On the left, Romanowski stain showing numerous alveolar macrophages with abundant vacuolated cytoplasm. On the right, positive orangestained droplets within the cytoplasmic vacuoles of alveolar macrophages.

test were negative. Urine pregnancy test was negative. Urine drug screen was positive for cannabinoid.

Chest $x$-ray on initial evaluation revealed diffuse patchy bilateral opacities with no effusions. Right upper quadrant ultrasound was normal.

Patient was admitted and placed on azithromycin and ceftriaxone for community-acquired pneumonia. Subsequently was changed to levofloxacin $750 \mathrm{mg}$ oral. Due to continued oxygen desaturation on ambient air, intermittent fevers, and tachycardia for two-day duration despite antibiotics and supportive care a high-resolution computed topography (HRCT) of the chest was obtained.

HRCT demonstrated widespread ground glass opacities and scattered septal thickening bilaterally without lobar predominance, with diffuse subpleural sparing (Figure 1). There were dense consolidations seen in the bilateral lobes and the right middle lobe. These findings were consistent with inhalation-induced lung injury as well as viral and atypical pneumoniae.

Patient subsequently underwent bronchoscopy. Findings notable for normal airway with parenchymal infiltrates. Bronchoalveolar lavage (BAL) with a transbronchial biopsy were performed. Right middle and left upper lobe BAL pathology revealed oil-red $O$ stain showing intracytoplasmic lipid droplets in alveolar macrophages consistent with lipid laden-macrophages (Figure 2). Transbronchial biopsy of the left lingula showed pneumonitis; there was interstitial edema, inflammation, mild fibrosis, hyperplasia of type II pneumocytes, organizing pneumonia, and macrophages in the airspace.

The history, imaging and pathology were felt to be consistent with EVALI. Due to this the patient was placed on three days of methylprednisone 250 mg every 12 hours. Upon the second day of this regimen, she no longer had ambulatory desaturation with stable vital signs. She finished a seven-day course of antibiotics while hospitalized. She was discharged on $40 \mathrm{mg}$ daily of prednisone for with subsequent pulmonary medicine outpatient visit, which has not yet occurred.

\section{DISCUSSION}

EVALI is lung injury due to the inhalation of non-combustible aerosol containing "nicotine, flavors, propylene glycol, and vegetable glycerin", which includes battery operated devices that include electronic cigarettes (e-cigarettes). ${ }^{1}$ EVALI is becoming a more prevalent, being formally recognized in 2019, with over 2,000 cases reported in the United States by the Center for Disease Control (CDC).2.3 This new clinical entity has led to interest in the pathogenesis of EVALI. Though no single agent has been implicated in the pathogenesis of EVALI, many patients have used nicotine, tetrahydrocannabinol (THC), and cannabinoid (CBD) oils.2.4 Another agent of interest implicated in in the pathogenesis of EVALI is vitamin E acetate, which has been associated with impairment of pulmonary surfactant. 5.6

EVALI is a type of acute lung-injury presenting with a wide-range of pulmonary complaints and findings. The most common presenting symptoms fevers, tachycardiac, and tachypnea. ${ }^{4}$ One review revealed gastrointestinal symptoms in about $80 \%$ of patients. In this same review, $67 \%$ of patients presented with hypoxemia, and about $30 \%$ of patients had oxygen saturation less than $88 \% .{ }^{4}$ Chest imaging generally reveals marked bilateral opacities. The typical chest CT finding is ground glass opacities marked by subpleural sparing ${ }^{4.7}$ These findings, however, can be seen in other pathological process and thus may warrant 


\section{Fonseca-Paricio, MD et al.: Case Report of E-cigarette Associated Lung Injury}

further investigations with bronchoscopy and BAL. EVALI leads to alveolar damage and fibrinous pneumonitis and has a wide range of histopathological findings. Most reported cases involve variation in cell counts from BAL, though usually with a predominance of neutrophils. ${ }^{4}$ Many cases report Oil-red-O staining lipid-laden macrophages as in this patient. ${ }^{8}$

One confounding variable in this case was a positive rhinovirus/enterovirus test, which could explain some of her symptoms. However, it is unlikely that this viral infection would have produced such significant clinical decompensation in an otherwise young and healthy patient. Moreover, clinical history, ground glass opacities with subpleural sparing, and lipid-laden macrophages with pneumonitis are consistent with our current understanding of EVALI.

\section{CONCLUSION}

In patients who present with respiratory complaints and history of E-cigarettes, EVALI should be strongly considered. This is particularly true in those with hypoxemia and respiratory distress. A chest CT is an Important diagnostic modality to help identify and inhalation pattern injury, but many patients may need further testing with bronchoscopy and BAL. We presented a case of a young, healthy patient who had significant respiratory distress secondary to EVALI.

\section{REFERENCES}

1. Gotts JE, Jordt SE, McConnell R, Tarran R. (2019). What are the respiratory effects of e-cigarettes? The BMJ, 366: 15275 .

2. Schier JG, Meiman JG, Layden J, et al. (2019). Severe Pulmonary Disease Associated with Electronic-Cigarette-Product Use - Interim Guidance. MMWR Morbidity and Mortality Weekly Report 2019, 68:787-790.

3. Chatham-Stephens K, Roguski K, Jang Y, et al. (2019). Characteristics of Hospitalized and Nonhospitalized Patients in a Nationwide Outbreak of E-cigarette, or Vaping, Product Use-Associated Lung Injury - United States, November 2019. MMWR Morbidity and Mortality Weekly Report 2019, 68:1076-1080

4. Layden J, Ghinai I, Pray I, et al. (2019). Pulmonary Illness Related to E-Cigarette Use in Illinois and Wisconsin - Preliminary Report. New England Journal of Medicine. Sept 6, 2019.

5. Blount BC, Karwowski MP, Morel-Espinosa M, et al. (2019). Evaluation of Bronchoalveolar Lavage Fluid from Patients in an Outbreak of E-cigarette, or Vaping, Product Use-Associated Lung Injury - 10 States, August-October 2019. MMWR Morbidity and Mortality Weekly Report 2019, 68:1040-1041.

6. Casals C, Cañadas O. (2012). Role of lipid ordered/disordered phase coexistence in pulmonary surfactant function. Biochimica et Biophysica Acta. 1818: 2550-2562

7. Henry TS, Kanne JP, Kligerman SJ. (2019). Imaging of vaping-associated lung disease. New England Journal of Medicine. 381:1486-1487.

8. Maddock SD et al. (2019). Pulmonary Lipid-Laden Macrophages and Vaping New England Journal of Medicine. 381.15:1488. [e-pub]. 